\title{
Continuous Orientation Representation for Arbitrary Dimensions - A Generalized Knutsson Mapping
}

\author{
Bernd Rieger ${ }^{\star}$, Lucas J. van Vliet, and Piet W. Verbeek \\ Quantitative Imaging Group, \\ Delft University of Technology, \\ Lorentzweg 1, 2628 CJ Delft, The Netherlands \\ \{b.rieger, l.j.vanvliet, p.w.verbeek\}@tudelft.nl
}

\begin{abstract}
In this paper we present a framework to construct a continuous orientation representation in arbitrary dimensions. Existing methods for 2D (doubling the angle) and 3D (Knutsson mapping) were found ad hoc. We show how they can be put in a general framework to derive suitable representations for filtering in spaces of arbitrary dimension. The dimensionality of the derived representation is shown to be minimal. Connections with the gradient structure tensor and Knutsson mapping are shown, like the fact that angle doubling works in each pair-cone of the Knutsson mapping. Finally, using projection operators we show how angles between vectors in the base space are related to vectors in the mapped spaces and in particular how to achieve preservation of isotropy.
\end{abstract}

Keywords: orientation representation, angle doubling, projection operators.

\section{Introduction}

The representation of directional information such that filtering can be applied without discontinuities has received considerable attention in the early days of image analysis for two-dimensional images [1,34. Later representations for 3-dimensional images have been found [56]. Most notably are here the contributions of Knutsson [45], who found a 5D minimal representation for 3D data and Granlund who found the angle doubling for 2D data [1].

The important role of directional information comes from the need to characterize simple neighborhoods - the local structure - in images. They are the basic building blocks of images. Simple neighborhoods are commonly referred to as shift invariant in at least one direction and not shift invariant in at least one other direction 2 37]. First order intensity variations are described by gradients and a collection of local gradients is needed to compute a dominant orientation. A vector has direction but locally only the orientation is needed. Representing orientation by angles (direction information) is troublesome, in the sense that it

^ Corresponding author.

A. Heyden and F. Kahl (Eds.): SCIA 2011, LNCS 6688, pp. 774 783, 2011.

(C) Springer-Verlag Berlin Heidelberg 2011 
is discontinuous. E.g. representing a line in $2 \mathrm{D}$ by its angle $[0,2 \pi]$ with respect to a fixed coordinate axis is not suitable. More general, vectors $v$ and $-v$ have the same orientation but point in opposite directions.

Averaging operations on directional representation by standard filtering in order to reduce noise produce artifacts at the discontinuities given by e.g. the angle representation. Standard filtering requires a continuous orientation representation, i.e. to compute the local dominant orientation. A well-known tool to analyze one-dimensional neighborhoods from gradients is the Gradient Structure Tensor (GST) [2/3/8,9/0]. It is defined as the dyadic product of the image gradients

$$
\bar{G}:=\overline{\nabla I \nabla I^{t}} \text { and } G:=\nabla I \nabla I^{t},
$$

where $I$ is a $n \mathrm{D}$ grey-value image and the over-lining stands for averaging the tensor elements inside a local neighborhood. This smoothing scale is typically 3 to 10 times larger than the scale at which the (regularized) gradients are computed. This suppresses gradient orientation contributions due to noise and yields a smooth, robust orientation output. Interpretation of the filtering is done via an eigenvalue analysis of $G$ and the ratio of the eigenvalues describe the local structure. Other applications that require continuous orientation representations include the estimation of curvature (i.e. the rate of change of local orientation) 1112 and block matching in MRI diffusion tensor imaging [13. Also the widely used Hough transform for detecting straight lines benefits from a continuous parameter representation [1415.

For locally multi-directional neighborhoods more complex approaches are needed. Structure such as crossing fibers or corners in images have locally more than one dominant orientation. The eigenvalue analysis of e.g. the structure tensor cannot handle that and standard smoothing of the representation will not return any of the dominant orientations, but a weighted average. A variety of different approaches have been developed that can deal with these neighborhoods 16171819 .

In 1989 Knutsson [5] introduced the following mapping $M: \mathbb{R}^{n} \rightarrow \mathbb{R}^{n \times n}$ to map discontinuous to continuous representations that allow further processing

$$
M(v)=\frac{v v^{t}}{\|v\|}, v \in \mathbb{R}^{n} .
$$

Here the vector $v$ is generally the image gradient. From the construction it is clear that $M$ is symmetric and has only $n(n+1) / 2$ independent components. The mapping is slightly different from the structure tensor $G$. Both mappings treat antipodal vectors the same by mapping them onto the same tensor $M(v)=M(-v)$. They have a rotation invariant norm, i.e. the information carried by the magnitude is not mixed with information carried by the angle (polar separability). However, eq. 2 differs from the GST in that it preserves distances between the input and output space (uniform stretch), i.e. $\|\delta M(v)\|=c\|\delta v\|$ for $\|v\|=$ const. whereas $\|\delta G(v)\|=c\|\delta v\|\|v\|$. The uniform stretch property allows to compute curvature of lines and surfaces in $n \mathrm{D}$ by applying derivative filters to $M$ and to interpret the outcome [1112]. Without distance preservation the outcome of 
derivative filters on the mapped representation cannot be interpreted in the same way as in the base space.

The property of a rotation invariant norm of eq. 2] leads to another restriction on the mapping in the form of $\operatorname{trace}(M)=$ const.. Therefore the dimensionality of the mapping proposed in eq. 2 can be further reduced [6], i.e. in addition to omitting the non-unique terms. Applying this dimensionality reduction to the $2 \mathrm{D}$ case of eq. 2 in polar form leads to the double angle method of Granlund $r(\cos 2 \varphi, \sin 2 \varphi)[276$. Already in 1985 Knutsson presented the solution for the $3 \mathrm{D}$ case [4] ad hoc. Later it turned out that his solution can also be directly obtained from eq. 2 by applying dimensionality reduction and reverting to polar coordinates [6].

\section{Construction of the General Knutsson Mapping}

The construction of the mapping with minimal dimensions from eq. 2 for the n-dimensional case has remained unclear. In [6] we already observed that the dimensionality reduction of eq. 2 led to the angular harmonics in 2D and the spherical harmonics in 3D. The construction, however, of an orthonormal basis for higher dimensional spherical harmonics is not straight-forward [20].

Here we present a concept to iteratively construct an orthonormal basis to represent orientation in $n \mathrm{D}$. We put the dimensionality reduction into a framework that allows intuitive construction. Let the diagonal elements $\left\{M_{i i}\right\}, i=1, \ldots n$

of eq. 2 span a $n \mathrm{D}$ space where $M_{i i}$ is one basis vector $(0, \ldots, 1,0, \ldots, 0)$. As the trace must be constant, i.e. $\sum M_{i i}=$ const., we rotate the basis such that one new axis is aligned with $\mathbf{1}=(1, \ldots 1)$ and the rest are orthogonal to it (orthonormality can always be achieved later if necessary by normalization). The off-diagonal elements of eq. 2 remain untouched, they comprise already a minimal set, and will flow into the mapping as is.

\subsection{The 2D Case in the General Framework}

We now apply a (unknown) rotation matrix $\mathbf{R}_{\mathbf{2}}$ to the standard basis $\mathbf{I}$ in $2 \mathrm{D}$ such that $\mathbf{R}_{\mathbf{2}} \mathbf{I}=\mathbf{D}_{\mathbf{2}}$ produces a matrix $\mathbf{D}_{\mathbf{2}}$ in which the last column is a vector with only ones $\mathbf{1}=(1,1)$. For the $2 \mathrm{D}$ case this recipe looks for a rotation matrix $\mathbf{R}_{2}$ :

$$
\mathbf{R}_{\mathbf{2}}\left(\begin{array}{ll}
1 & 0 \\
0 & 1
\end{array}\right)=\left(\begin{array}{ll}
a_{1} & 1 \\
a_{2} & 1
\end{array}\right)=\mathbf{D}_{\mathbf{2}}
$$

such that the inner product of vector $a$ and $\mathbf{1}$ is zero, i.e. $(a, \mathbf{1})=\left(a_{1}, a_{2}\right) \cdot(1,1)=$ $0 \Rightarrow a_{1}=-a_{2}$. Up to a scaling factor the matrix $\mathbf{D}_{\mathbf{2}}$ is again a rotation matrix. Let us choose $a_{1}=1$ and apply $\mathbf{D}_{2}$ to the diagonal elements of $M(v)=\left(v_{1}^{2}, v_{2}^{2}\right)$ for $\|v\|=r=1$ and we obtain $v^{T} \boldsymbol{D}_{2}=\left(v_{1}^{2}-v_{2}^{2}, v_{1}^{2}+v_{2}^{2}=\right.$ const. $)=$ ( $\cos 2 \varphi$, const.). Together with the off-diagonal terms $2 v_{1} v_{2}=\sin 2 \varphi$ and normalization with $\|v\|=r$ this gives indeed the well-known double angle representation 112. The connection between the tensor representation of eq. 2 and the double angle method has already been pointed out and made explicit in [7, Chapter 9]. 


\subsection{The 3D Case in the General Framework}

Going to 3D we can use the 2D result if we rotate only in subspaces orthogonal to the ones constructed for $2 \mathrm{D}$. This way we construct $\mathbf{D}_{\mathbf{3}}$

$$
\mathbf{R}_{3}\left(\begin{array}{lll}
1 & 0 & 0 \\
0 & 1 & 0 \\
0 & 0 & 1
\end{array}\right)=\left(\begin{array}{rrr}
1 & b_{1} & 1 \\
-1 & b_{2} & 1 \\
0 & b_{3} & 1
\end{array}\right)=\mathbf{D}_{\mathbf{3}}
$$

such that $b \perp \mathbf{1} \perp(1,-1,0)$. This orthogonality gives two equations for the components of vector $b$

$$
\begin{aligned}
b_{1}-b_{2} & =0 \\
b_{1}+b_{2}+b_{3} & =0 .
\end{aligned}
$$

Let us choose $b_{1}=-1 \Rightarrow b_{2}=-1, b_{3}=2$. This choice yields the mapping Knutsson found intuitively 4. Applying this choice of $\mathbf{D}_{\mathbf{3}}$ to the diagonal elements of $M(v)=\left(v_{1}^{2}, v_{2}^{2}, v_{3}^{2}\right)$ for $\|v\|=1$ we obtain $v^{T} \mathbf{D}_{\mathbf{3}}=\left(v_{1}^{2}-v_{2}^{2}, 2 v_{3}^{2}-\right.$ $v_{1}^{2}-v_{2}^{2}, v_{1}^{2}+v_{2}^{2}+v_{3}^{2}=$ const. $)$. In polar form this reads $\left(\sin ^{2} \vartheta \cos 2 \phi, \sqrt{3}\left(\cos ^{2} \vartheta-\right.\right.$ $\left.\frac{1}{3}\right)$, const.). Putting these terms together with the off-diagonal elements $\left(v_{1} v_{2}, v_{1} v_{3}, v_{2} v_{3}\right)$ of eq. 2 we arrive at the original description by Knutsson [6].

\subsection{The $n \mathrm{D}$ Case in the General Framework}

We notice that there was a choice in one of the components of the vector $a$ and $b$ to fulfill the requirements of orthogonality. This implies of course that the mappings are not unique in terms of scaling (of an axis) as long as we do not require orthonormality. We will also see that the implicit historic choices for $a$ and $b$ do not generalize well to $n \mathrm{D}$. For a systematic iterative construction let us chose $a_{1}=-1 \Rightarrow a_{2}=1$, or

$$
\mathbf{D}_{2}=\left(\begin{array}{rr}
1 & 1 \\
-1 & 1
\end{array}\right)
$$

and for $3 \mathrm{D}$ we use the earlier $b_{1}=-1 \Rightarrow b_{2}=-1, b_{3}=2$, or

$$
\mathbf{D}_{\mathbf{3}}=\left(\begin{array}{rrr}
-1 & -1 & 1 \\
1 & -1 & 1 \\
0 & 2 & 1
\end{array}\right) \text {. }
$$

From this we can continue the construction for $n \mathrm{D}$ as follows

$$
\mathbf{D}_{\mathbf{n}}=\left(\begin{array}{rrrrr}
-1 & -1 & -1 & \cdots & 1 \\
1 & -1 & -1 & \cdots & 1 \\
0 & 2 & -1 & \cdots & 1 \\
0 & 0 & 3 & \cdots & 1 \\
0 & 0 & 0 & \ddots & 1 \\
0 & 0 & 0 & -1 & 1
\end{array}\right)
$$


The last column vector is $\mathbf{1}$ and the $i$-th vector is $\overbrace{-1, \ldots,-1}^{i \text { times }}, i, 0, \ldots)$. It is apparent from this iterative construction that all properties for each subspace are preserved. That is, the lower dimensional mappings are embedded in the higher dimensional representation. For example application of $\mathbf{D}_{\mathbf{4}}$ to the diagonal elements of $M(v)$ in $4 \mathrm{D}$ reduces the set as follows: $\left(v_{1}^{2}, v_{2}^{2}, v_{3}^{2}, v_{4}^{2}\right) \rightarrow\left(-v_{1}^{2}+\right.$ $v_{2}^{2},-v_{1}^{2}-v_{2}^{2}+2 v_{3}^{2},-v_{1}^{2}-v_{2}^{2}-v_{3}^{2}+3 v_{4}^{2}$, const.). Orthonormality can always be imposed if desired.

\section{Alternative Mapping Based on Projection Operators and Angles between Vectors in Mapped Space}

We shall now give an alternative mapping based on projection operators of the form $I-P_{I}$. This mapping gives the same angles between mapped vectors and therefore must be equivalent to the reduced mappings (angle doubling, 4, Eq. 50] and the general mapping implied by eq. 9), i.e. equal up to a global rotation in mapped space. This rotation implicit in the former mappings brings the identity matrix along one of the axes (the last variable indicated as const.). In the alternative presented here we just project it away.

A projection of a vector $w$ onto a vector $v$ is given by $P_{v} w=\frac{(v, w)}{(v, v)} v$, with $v, w, \in \mathbb{R}^{n} . P_{v}$ is idempotent and we have $\left(I-P_{v}\right) P_{v}=P_{v}\left(I-P_{v}\right)=0$. Using this property the inner product of two vectors $v, w$ can be split into $(v, w)=$ $\left(P_{a} v, P_{a} w\right)+\left(\left(I-P_{a}\right) v,\left(I-P_{a}\right) w\right)$ by projection onto an arbitrary vector $a \in \mathbb{R}^{n}$. The projection onto $I-P_{a}$ is to be interpreted as the projection onto the $n-1$ dimensional hyperplane with normal $a$.

The angle between two vectors in mapped space by projections (from eq. 2) is in operator notation $\cos \angle\left(P_{v}, P_{w}\right)=\left(P_{v}, P_{w}\right)=\cos ^{2} \angle(v, w)=\frac{1}{2}+$ $\frac{1}{2} \cos (2 \angle(v, w))$. This result was already found in [6, Eq. 21].

We now propose the following equivalent alternative mapping to the iterative construction of eq. 9

$$
v \rightarrow\left(I-P_{I}\right) P_{v} .
$$

To deduce the angle between the projection operators $\left(I-P_{I}\right) P_{v}$ and $\left(I-P_{I}\right) P_{w}$ we use eq. 24 from the appendix

$$
\begin{aligned}
\cos \angle\left(\left(I-P_{I}\right) P_{v},\left(I-P_{I}\right) P_{w}\right) & =\frac{\cos \angle\left(P_{v}, P_{w}\right)-n^{-1}}{1-n^{-1}} \\
& =\frac{1-2 n^{-1}}{2-2 n^{-1}}+\frac{\cos (2 \angle(v, w))}{2-2 n^{-1}}
\end{aligned}
$$

where we have used

$$
\cos \angle\left(I, P_{v}\right)=\frac{\left(I, P_{v}\right)}{\|I\|\left\|P_{v}\right\|}=\frac{1}{\sqrt{n}}
$$


If we make this relation explicit for $n=2,3,4$ we get for eq. 12

$$
\begin{array}{ll}
n=2: & 0+\cos (2 \angle(v, w)), \\
n=3: & \frac{1}{4}+\frac{3}{4} \cos (2 \angle(v, w)), \\
n=4: & \frac{1}{3}+\frac{2}{3} \cos (2 \angle(v, w)) .
\end{array}
$$

The case $n=2$ was already long known as doubling of the angle and gives the same relation as eq. 3. The case $n=3$ gives the same relation as the mapping of Knutsson as in [4, Eq. 50] or of course as eq. 8. For all higher dimensional mappings generated by eq. 9 the angles in mapped space are to be computed by eq. 12. For example for $n=3$ we get for the diagonal elements by eq. 8 $\left(-v_{1}^{2}+v_{2}^{2},-v_{1}^{2}-v_{2}^{2}+2 v_{3}^{2}, v_{1}^{2}+v_{2}^{2}+v_{3}^{2}=\right.$ const.) (up to scaling) and using the projection of eq. 10] we get $\frac{1}{3}\left(2 v_{1}^{2}-v_{2}^{3}-v_{3}^{2},-v_{1}^{2}+2 v_{2}^{2}-v_{3}^{2},-v_{1}^{2}-v_{2}^{2}+2 v_{3}^{2}\right)$. See also the appendix for a comparison of the mappings.

From eq. 13 we see that $1 / n$ can be interpreted as the average cosine angle between two vectors in the mapped space $M$ or

$$
\left\langle\cos \angle\left(P_{v}, P_{w}\right)\right\rangle=\left\langle\cos ^{2} \angle(v, w)\right\rangle=\frac{1}{n} .
$$

In this average, the vector $v$ is fixed and the set $\{w\}$ has uniform distribution over the $n-1$ dimensional solid angle.

From here it is not yet clear what the advantage is to use $v \rightarrow\left(I-P_{I}\right) P_{v}$ instead of the approach $v \rightarrow P_{v}$ of eq. 2, however, in the following we will see that if we want the mapping to preserve the isotropy then it is indeed useful. Eq. 9 is also shown to preserve isotropy and reduces the components of the mapping to be computed by one compared to eq. 2 or eq. 10.

\section{Preserving Isotropy in Mapped Space}

Summarizing the relations between angles in mapped space and original space (eq. 11] and 13) we get

$$
\begin{aligned}
\cos \angle\left(\left(I-P_{I}\right) P_{v},\left(I-P_{I}\right) P_{w}\right) & =\frac{\cos \angle\left(P_{v}, P_{w}\right)-\left\langle\cos \angle\left(P_{v}, P_{w}\right)\right\rangle}{1-\left\langle\cos \angle\left(P_{v}, P_{w}\right)\right\rangle} \\
& =\frac{\cos (2 \angle(v, w))-\langle\cos (2 \angle(v, w))\rangle}{1-\langle\cos (2 \angle(v, w))\rangle} .
\end{aligned}
$$

Now we see that the average cosine angle of this expression is zero, i.e. $\left\langle\cos \angle\left(\left(I-P_{I}\right) P_{v},\left(I-P_{I}\right) P_{w}\right)\right\rangle=0$, just as is the case for the average angle $\langle\cos \angle(v, w)\rangle$. Using the operator $\left(I-P_{I}\right) P_{v}$ produces a representation that preserves isotropy.

The angle between operators does not change when they are multiplied by a positive scalar $\angle\left(P_{v}^{\prime}, P_{w}^{\prime}\right)=\angle\left(P_{v}, P_{w}\right)$ with $P_{v}^{\prime}=f(v) P_{v}$. Moreover, if $f(v)$ does not depend on the direction of $v$, preservation of isotropy (the zero average of eq. 15) also holds for $P^{\prime}$ instead of $P$. Now we can characterize Knutsson's 
mapping and our iterative construction scheme as follows: i) [4, Eq. 18-22] for $3 \mathrm{D}$ is equivalent to isotropy preservation of $\|v\|\left(I-P_{I}\right) P_{v}$, ii) [5, Eq. 4] (or eq. 2) for $n \mathrm{D}$ is equivalent to $\|v\| P_{v}$ and iii) the iterative construction for $n \mathrm{D}$ of eq. 9 is equivalent to isotropy preserving $\|v\|\left(I-P_{I}\right) P_{v}$.

Please note, that Knutsson always used $f(v)=\|v\|$. We remark that mappings with different choices of $f(v)$ could be studied, e.g. $f(v)=\|v\|^{p}$. In particular $p=0$ and $p=2$ look interesting, with $D_{v}=\|v\|^{2} P_{v}$ being the dyadic product of $v$ and $v$ applied in the gradient structure tensor.

\section{Doubling the Angle - Knutsson Cones in Higher Dimensions}

The angle between two mapped vectors has been investigated by Knutsson with special attention to the case of a 2D Euclidean subspace (plane) in 3D [4. He found that each halfplane in his $5 \mathrm{D}$ reduced mapping is mapped onto a $2 \mathrm{D}$ manifold in a separated 3D subspace where the manifold is the surface of a cone. Note that doubling of the half-plane angle occurs in mapping the half-plane onto the surface of a cone. That is due to the fact that the iterative construction of the generalized mapping for arbitrary dimensions embeds all lower dimensional mappings with their properties among which the angle doubling that was found in $2 \mathrm{D}$.

In generalizing from $2 \mathrm{D}$ to $n \mathrm{D}$ one might hope for generalization of the cone to a hypercone. That appears to be impossible. Each pair of axes in $n \mathrm{D}$ forms a $2 \mathrm{D}$ subspace with a corresponding cone. The extra dimension of a cone represents at once both off-diagonal elements in the symmetric matrix. In $n \mathrm{D}$ the number of off-diagonal element pairs can only be represented by one extra dimension for each $2 \mathrm{D}$ subspace and this is what one cone per $2 \mathrm{D}$ subspace amounts to. In Fig. 1 and Fig. 2 we visualize the cases for $n=2$ and $n=3$; below we formalize our reasoning.

Let us consider now for sake of simplicity, but without loss of generality a normal vector $v=\left(c_{1}, c_{2}, c_{3}\right) \in \mathbb{R}^{3}$ with $\|v\|=1$, where $c_{i}$ represents the
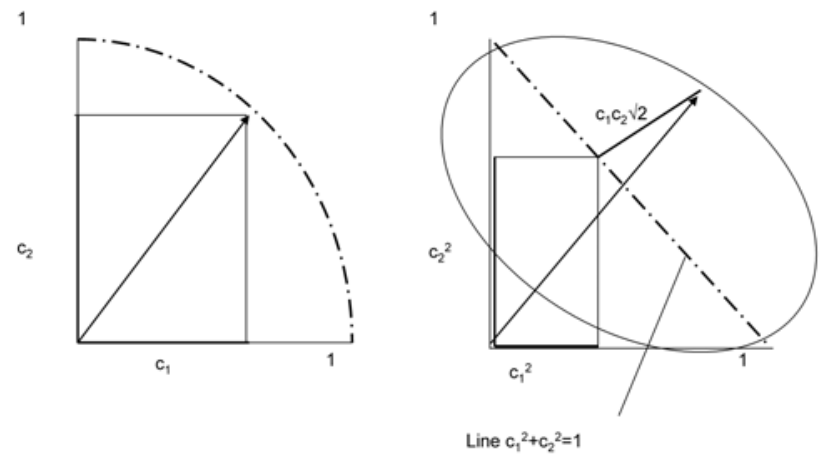

Fig. 1. For the case $n=2$ we draw the relation between the diagonal elements $c_{1}^{2}, c_{2}^{2}$ and the off-diagonal element $\sqrt{2} c_{1} c_{2}$ and how this pair forms a cone 


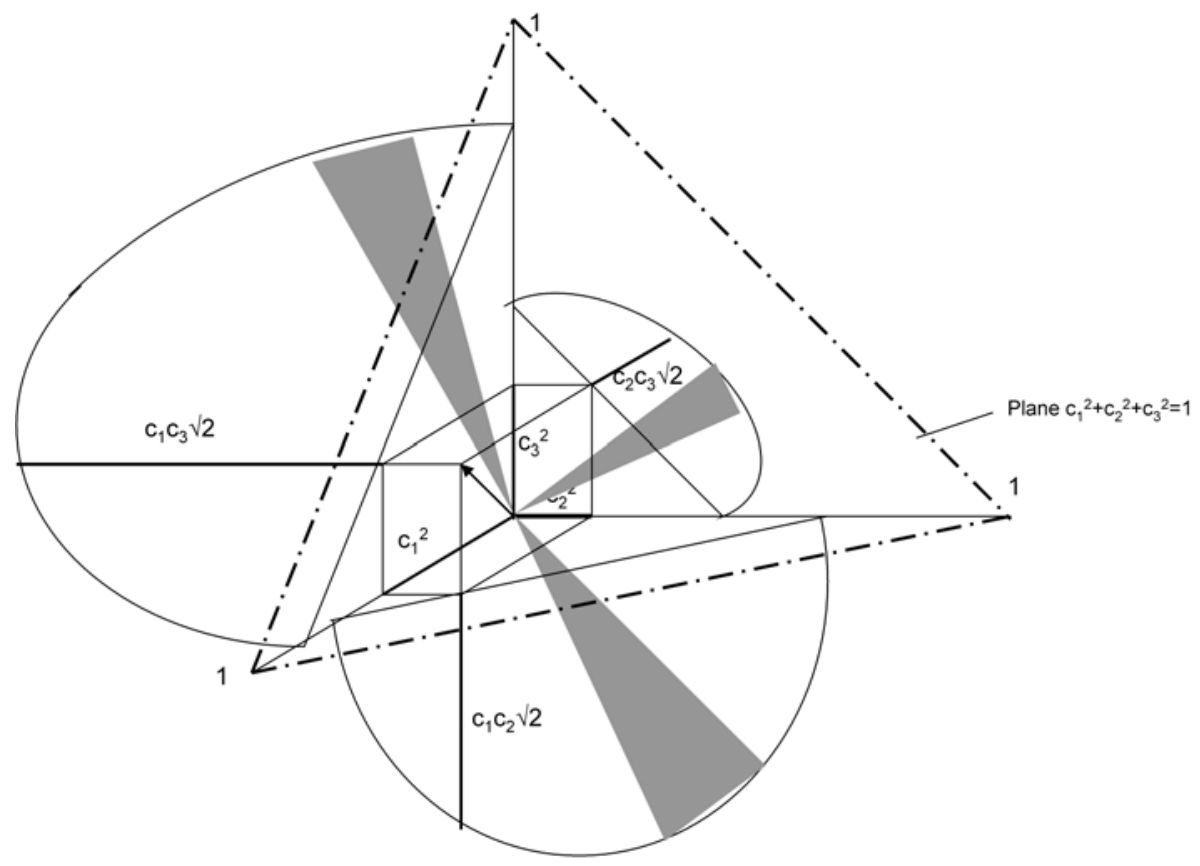

Fig. 2. Knutsson cones in higher dimensions. In the general case each pair of $c_{i}, c_{j}$ gives one extra dimension and one guiding cone. The extra dimension of a cone represents at once both off-diagonal elements in the symmetric matrix.

direction cosine. For the diagonal components of $M(c)$ is it clear that they stay in the subspace $c_{1}^{2}+c_{2}^{2}+c_{3}^{2}=1$. The non-diagonal elements $c_{1} c_{2}, c_{1} c_{3}, c_{2} c_{3}$ follow the respective guiding cones, i.e. the angle doubling works per pair-cone. Also in higher dimensions only pairs operate in 2D. For the cones we must check the norms such that everything adds up to one. This will give a scaling factor of $\sqrt{2}$ for the $n(n+1) / 2$-dimensional mapping $M$

$$
\begin{aligned}
c_{1}^{2}+c_{2}^{2}+c_{3}^{2} & =1 \\
\left(c_{1}^{2}\right)^{2}+\left(c_{2}^{2}\right)^{2}+\left(c_{3}^{2}\right)^{2}+\left(\sqrt{2} c_{1} c_{2}\right)^{2}+\left(\sqrt{2} c_{1} c_{3}\right)^{2}+\left(\sqrt{2} c_{2} c_{3}\right)^{2} & =1 .
\end{aligned}
$$

\section{Conclusion}

This paper presents a generalized framework for the construction of a continuous orientation representation in arbitrary dimensions. The mappings for all dimensions lower than the current one are embedded and hence its properties hold as well. We introduced projection operators as an alternative to construct isotropy preserving mappings. The ad hoc constructions of Granlund and Knutsson are now explicit and available for arbitrary dimensional spaces. The well-known angle doubling works in each pair-cone of the Knutsson mapping. 


\section{References}

1. Granlund, G.H.: In search of a general picture processing operator. Computer Graphics and Image Processing 8, 155-173 (1978)

2. Bigün, J., Granlund, G.H.: Optimal orientation detection of linear symmetry. In: Proceedings of the First IEEE International Conference on Computer Vision, London, pp. 433-438. IEEE Computer Society Press, Los Alamitos (1987)

3. Kass, M., Witkin, A.: Analyzing oriented patterns. Computer Vision, Graphics and Image Processing 37, 362-385 (1987)

4. Knutsson, H.: Producing a continuous and distance preserving 5-d vector representation of 3-d orientation. In: IEEE Computer Society Workshop on Computer Architecture for Pattern Analysis and Image Database Management, Miami Beach, Florida, pp. 175-182 (1985)

5. Knutsson, H.: Representing local structure using tensors. In: The 6th Scandinavian Conference on Image Analysis, Oulu, Finland, pp. 244-251 (1989)

6. Rieger, B., van Vliet, L.J.: A systematic approach to $\mathrm{nD}$ orientation representation. Image and Vision Computing 22, 453-459 (2004)

7. Granlund, G.H., Knutsson, H.: Signal processing for computer vision. Kluwer Academic Publishers, Boston (1995)

8. Jähne, B.: Digital Image Processing, 4th edn. Springer, Berlin (1997)

9. van Ginkel, M., van de Weijer, J., Verbeek, P.W., van Vliet, L.J.: Curvature estimation from orientation fields. In: Ersboll, B.K., Johansen, P. (eds.) SCIA 1999, Proc. 11th Scandinavian Conference on Image Analysis, Kangerlussuaq, Greenland, pp. 545-551. Pattern Recognition Society of Denmark, Lyngby (1999)

10. Bigün, J., Granlund, G.H., Wiklund, J.: Multidimensional orientation estimation with applications to texture analysis and optical flow. IEEE Transactions on Pattern Analysis and Machine Intelligence 13, 775-790 (1991)

11. Rieger, B., van Vliet, L.J.: Curvature of n-dimensional space curves in grey-value images. IEEE Transactions on Image Processing 11, 738-745 (2002)

12. Rieger, B., van Vliet, L.J., Verbeek, P.W.: Estimation of curvature based shape properties of surfaces in 3D grey-value images. In: Bigun, J., Gustavsson, T. (eds.) SCIA 2003. LNCS, vol. 2749, pp. 262-267. Springer, Heidelberg (2003)

13. van Noorden, S., Caan, M.W.A., van der Graaf, M., van Vliet, L.J., Vos, F.M.: A comparison of the cingulum tract in ALS-B patients and controls using kernel matching. In: Jiang, T., Navab, N., Pluim, J.P.W., Viergever, M.A. (eds.) MICCAI 2010. LNCS, vol. 6362, pp. 249-256. Springer, Heidelberg (2010)

14. Westin, C.-F., Knutsson, H.: The Möbius strip parameterization for line extraction. In: Sandini, G. (ed.) ECCV 1992. LNCS, vol. 588, pp. 33-38. Springer, Heidelberg (1992)

15. Westin, C.F.: A Tensor Framework for Multidimensional Signal Processing. PhD thesis, Linköping University, Linköping, Sweden (1994)

16. Mühlich, M., Aach, T.: Analysis of multiple orientations. IEEE Transactions on Image Processing 18, 1424-1437 (2009)

17. Franken, E.M., Duits, R., Haar Romenij, B.M.: Nonlinear diffusion on the 2D euclidean motion group. In: Sgallari, F., Murli, A., Paragios, N. (eds.) SSVM 2007. LNCS, vol. 4485, pp. 461-472. Springer, Heidelberg (2007)

18. Faas, F.G.A., van Vliet, L.J.: 3D-orientation space; filters and sampling. In: Bigun, J., Gustavsson, T. (eds.) SCIA 2003. LNCS, vol. 2749, pp. 36-42. Springer, Heidelberg (2003) 
19. Herberthsson, M., Brun, A., Knutsson, H.: Pairs of orientation in the plane. In: Proceedings of the SSBA Symposium on Image Analysis. SSBA (2006)

20. Stein, E., Stein, E.M., Weiss, G.: Introduction to Fourier Analysis on Euclidean Spaces. Princeton University Press, Princeton (1971)

\section{Appendix}

\section{Projection Operator Properties}

Let us consider the angle between $P_{a} v$ and $P_{a} w$ and $\left(I-P_{a}\right) v$ and $\left(I-P_{a}\right) w$, but first we compute the required inner products

$$
\begin{aligned}
\left(P_{a} v, P_{a} w\right) & =\left(\frac{(a, v)}{(a, a)} a, \frac{(a, w)}{(a, a)} a\right) \\
& =(a, v)(a, w)(a, a) \\
& =\|v\|\|w\| \cos \angle(a, w) \cos \angle(a, v), \\
\left(\left(I-P_{a}\right) v,\left(I-P_{a}\right) w\right)= & (v, w)-\left(P_{a} v, P_{a} w\right) \\
& =\|v\|\|w\|(\cos \angle(v, w)-\cos \angle(a, w) \cos \angle(a, v))
\end{aligned}
$$

For $v=w$ we get $\left(\left(I-P_{a}\right) v,\left(I-P_{a}\right) v\right)=\|v\||\sin \angle(a, v)|$. Finally we arrive at

$$
\begin{gathered}
\cos \angle\left(\left(I-P_{a}\right) v,\left(I-P_{a}\right) w\right)=\cos \angle(w, v)-\frac{\cos \angle(a, v) \cos \angle(a, w)}{|\sin \angle(a, v)||\sin \angle(a, w)|}, \\
\cos \angle\left(P_{a} v, P_{a} w\right)=\cos \angle(a, v) \cos \angle(a, w) .
\end{gathered}
$$

Eqs. 19 to 25 are also valid for the mapped vectors of $a, v, w$.

\section{Relation between Iterative (eq. 9) and Alternative Mapping (eq. 10)}

Let the symmetric matrices span the space $\mathcal{S}$ of dimensionality $n+\left(n^{2}-n\right) / 2$. Further the off-diagonal symmetric matrices the $\left(n^{2}-n\right) / 2$-dimensional space $\mathcal{N}$ and the diagonal matrices the $n$-dimensional space $\mathcal{D}$, where $\mathcal{N}$ and $\mathcal{D}$ are orthogonal. $\mathcal{D}$ consists of the unit matrix $\mathbf{I}$ and its orthogonal complement, the space $\mathcal{T}$, of traceless matrices. The difference between eq. 9 and eq. 10 is the treatment of the unit matrix $\mathbf{I}$. In both cases $v \in \mathbb{R}^{n}$ is mapped to $\mathcal{S}$ by projection on $\mathcal{S}$ of $v^{T} v$ (or a scaled version of it with $f(v)$ ). The projection onto $\mathcal{N}$ is also the same, just copying the matrix elements $v_{i} v_{j}$ for $i<j$. The projection onto $\mathcal{D}$ is different. Eq. 9 constructs an orthogonal basis in $\mathcal{D}$ consisting of $\mathbf{I}$ and a basis of $\mathcal{T}$ consisting out of matrices orthogonal to $\mathbf{I}$. Then it is projected onto this basis in $\mathcal{D}$ and the projection onto $\mathbf{I}$ can be omitted as it is irrelevant for the goal of the mapping. Eq. 10 also copies the matrix elements $v_{i} v_{i}$ but eliminates the projection onto $\mathbf{I}$.

Eq. 10] uses $n$ components with condition that the sum over all components must be zero, where eq. 9. uses the condition that the last component must be constant. 